\title{
MOVERS
}

\section{Vasilis Ntziachristos, director, Institute for Biological and Medical Imaging, GSF, Neuherberg, Germany}

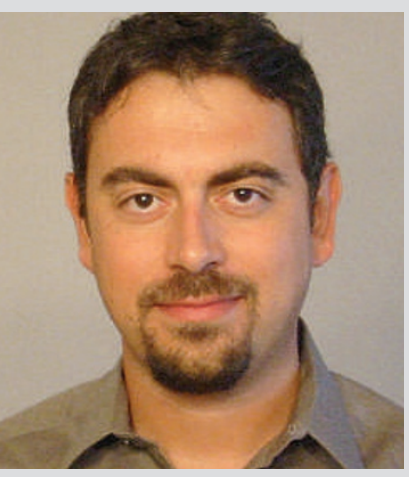

2002-07: Assistant professor, Harvard University Medical School and Massachusetts General Hospital, Boston, Massachusetts 2001-02: Instructor, University Medical School and Massachusetts General Hospital, Boston, Massachusetts

As an engineering undergraduate at the Aristotle University of Thessaloniki in his native Greece, Vasilis Ntziachristos was attracted to biomedical imaging as a way to use his skills to benefit others.

For his undergraduate thesis, he developed hardware and software platforms to improve the performance of magnetic resonance imaging (MRI). To get hands-on experience with imaging applications, Ntziachristos took a fellowship at the Panum Institute at the University of Copenhagen in Denmark. "It soon became apparent to me that I wanted to live at this interface between engineering, medicine and biology," he says.

One year later, he was a graduate student at the University of Pennsylvania in Philadelphia working with leading imaging scientist Britton Chance. There, Ntziachristos combined MRI with the emerging power of optical imaging to determine the molecular structure and function of tissues. The result was a new imaging approach to determine the molecular characteristics of breast cancer.

As a research fellow at Harvard Medical School in Boston, Ntziachristos continued to refine the optical technique to identify the molecular signatures of various tissues. Taking advantage of the concurrent explosion of genomic information, he developed techniques to decipher the molecular signatures of genes and proteins in vivo.

After seven years at Harvard, Ntziachristos is now moving on to a post in Germany. In November, he will take over as director of the Institute for Biological and Medical Imaging at the GSF-National Research Center for Environment and Health and will also become chair for the biological-imaging department at the Technical University of Munich. "Vasilis will help us visualize molecular events to better understand biology and pathology," says Gunther Wess, president of the GSF.

Ntziachristos has two major goals for the institute. He wants to create significantly better biological tools, and to move those tools into the clinic so that doctors can make more informed treatment decisions. His first challenge is building the multidisciplinary environment necessary for this work - he needs everything from chemistry expertise to skills in working with animal models - under one roof. Wess says it was Ntziachristos's collaborative zeal, as well as his technical expertise, that made him the perfect fit for such ambitions at the GSF.

Virginia Gewin
NETWORKS \& SUPPORT

\section{Brain work}

Jeff Hawkins got the neuroscience bug in 1979, but it took him many years and two inventions - the Palm Pilot and the Treo smartphone - to put his passion into action. His career arc illustrates how technological acumen and a theoretical mindset can foster one another: both his devices use pattern matching and computational decision-making algorithms that mimic theories of how a brain works.

As an undergraduate at Cornell University, Hawkins was impressed with a Scientific American article by Nobel laureate Francis Crick. "He was saying we have all these data about neurons and chemical processes in the brain and people act like they know what's going on, but don't believe it," Hawkins says. "What's lacking is a theoretical framework." Models were needed to show how the cells and processes interacted.

Hawkins suggested starting a neuroscience division at his first employer, Intel, but was rebuffed. He applied some of his theories about pattern recognition at Grid Systems in Sunnyvale, California, where he worked on handwriting recognition hardware and software. To learn more about how the brain processes pattern-matching, Hawkins went to the University of California, Berkeley, where he researched the workings of the brain's neocortex, which is associated with vision and decisionmaking. But he was told neocortical theory wasn't a viable thesis option.

That gave him insight into the obstacles of trying to develop an unfashionable field. "I learned that understanding the brain is not just a scientific problem, it's an institutional problem," Hawkins says.

His next few years were spent back at Grid, which later became Palm, developing Palm Pilot and Treo which he calls "sort of my day job, like an actor waiting on tables".

His success led him to start the Redwood Neuroscience Institute in Menlo Park, California, and co-write On Intelligence (Times Books, 2004), which expands on themes from his rejected thesis proposal. He later formed Numenta, which designs three-dimensional decision-making trees that can be applied to things from motion-capture video games to traffic sensors mounted on vehicles.

Hawkins thinks applications may drive more institutional change and private investment into neuroscience. "The commercial world is a very powerful way to get people to work on a problem," Hawkins says. "If you can show a profit, you can get a lot of

Paul Smaglik people to work on this."

\section{POSTDOC JOURNAL}

\section{Corporate America}

I recently contacted a former colleague who is now working in agro-industry. | am considering a career in industry, and I wanted to pick her brain for advice. She has worked in her position as group research leader for only a year, but she had some helpful insights. Although she has the job she wanted, the number of management duties has reduced her self-image as a serious scientist. Also, as she and her husband aren't able to live in the same state, it's nearly impossible for them to start a family. They've had trouble deciding who should give up their job to allow them to live together, even though she admits that this isn't her permanent career path.

That said, it's not all bad. Her company believes that leadership and scientific collaboration are important and encourages staff to attend training sessions, conferences and collaborative projects. Also, the pay is good, and the facilities help projects go faster and more smoothly than they might at a university.

Although not all research lends itself to a corporate output, I've come to realize that for many this is a good option. In particular, I like the way companies tend to be more family-friendly than universities - enabling employees to keep more regular hours, for example. For me, the hardest adjustment is the notion that science is profit, and that this has great influence on one's research. Moira Sheehan is a postdoc in the Department of Plant Breeding and Genetics at Cornell University. 